\title{
Modelagem dos efeitos do vento sobre as dimensões do alcance do jato de um canhão hidráulico ${ }^{1}$
}

\author{
Henrique F. E. de O liveira ${ }^{2}$, Alberto Colombo ${ }^{2}$ \& Lessandro C. Faria ${ }^{2}$
}

\begin{abstract}
RESUMO
A hipótese da existência de uma relação linear entre o raio de al cance de um canhão hidráulico e a velocidade do vento, que é assumida no model o semi-empírico de Richards \& W eatherhead, para simulação da distribuição de água de canhões hidráulicos operando sob condições de vento, foi avaliada neste trabalho. Na avaliação foram utilizadas distâncias observadas em ensaios de campo, entre o canhão Plona RL-250 e os limites da sua área molhada, tomadas nas seguintes direções: contrária, a favor e perpendicular ao sentido do vento. As retas ajustadas pelo método dos mínimos quadrados aos dados disponíveis, indicam que cada incremento de $1 \mathrm{~m} \mathrm{~s}^{-1}$ na velocidade do vento provoca as seguintes al terações, expressas em relação ao valor do raio sem vento do aspersor: (i) um decréscimo de $6,3 \%$ no sentido contrário ao vento $\left(R^{2}=0,737\right)$; (ii) um decréscimo de $7,3 \%$ nas direções perpendicular ao vento $\left(R^{2}=0,850\right)$; e (iii) um acréscimo de $1,3 \%$ no sentido do vento; esses resultados indicam que, no intervalo de velocidades de vento consideradas $\left(0\right.$ a $\left.5 \mathrm{~m} \mathrm{~s}^{-1}\right)$ a hipótese de uma relação linear entre raio de alcance e velocidade do vento pode ser utilizada.
\end{abstract}

Palavras-chave: irrigação, modelo semi-empírico, simulação

\section{Modeling of wind effects on dimensions of wetted area by a gun type sprinkler}

\begin{abstract}
The hypothesis of a linear relationship between wetted area of a sprinkler and the wind velocity which is assumed by the Richards \& W eatherhead semi empirical model for simulation of wind effects on gun type water application distribution was evaluated. Distances from the Plona-RL250 gun type sprinkler to the upwind, crosswind and downwind edges was determined in 53 field testes, held under different wind conditions and were used to evaluate the suitability of the proposed linear relationship. A linear least square fit for each set of data indicates that, for each $1 \mathrm{~m} \mathrm{~s}^{-1}$ increase in wind velocity, there is: (i) a $6.3 \%$ decrease on the distance to the upwind edge $\left(R^{2}=0.737\right)$; (ii) a $7.3 \%$ decrease on the distance to the crosswind edge $\left(R^{2}=0.850\right)$; and (iii) a $1.3 \%$ increase on downwind edge distance in relation to the no-wind radius of throw. These results indicate that, for the range of wind velocities considered in this study (from 0 up to $5 \mathrm{~m} \mathrm{~s}^{-1}$ ) the linear relationship between sprinkler reach and wind velocity can be accepted.
\end{abstract}

Key words: irrigation, semi empirical model, simulation 


\section{INTRODUÇÃO}

A desuniformidade de aplicação de água dos sistemas de irrigação reduz o retorno econômico que se pode obter em áreas irrigadas e aumenta o impacto ambiental desta atividade (Ortega et al., 2004), em função da redução na produtividade das culturas irrigadas e do desperdício de água, energia e fertilizantes.

O vento é, reconhecidamente, um dos fatores que mais afetam a uniformidade de aplicação de água dos sistemas de irrigação por aspersão. Azevedo et al. (2000), ao avaliarem o efeito de diferentes fatores sobre a uniformidade de aplicação de água de sistemas convencionais de aspersão, equipados com canhões hidráulicos, concluíram que a velocidade do vento foi o fator que mais influenciou a uniformidade de aplicação da água, seguida pela pressão de operação do aspersor, espaçamento entre aspersores na linha lateral, espaçamento entre linhas laterais, direção do vento em relação à linha lateral e velocidade de rotação do aspersor.

Em função da importância dos efeitos do vento sobre a uniformidade de aplicação de água dos sistemas de irrigação por aspersão e das dificuldades de se estudar esses efeitos em condições de campo, a modelagem em computador da distribuição de água de aspersores operando em condições de vento é muito utilizada.

Han et al. (1994) classificam os diversos modelos de simulação dos efeitos do vento sobre a distribuição espacial de água aplicada por aspersores em duas categorias: modelos físicos e modelos empíricos.

Os modelos físicos se baseiam na solução de sistemas de equações diferenciais que descrevem a trajetória de gotas desde o momento em que deixam o aspersor até o momento em que atingem a superfície do solo. Carrion et al. (2001) apresentam uma extensa lista de modelos físicos que simulam a distribuição espacial da água de aspersores operando em condições de vento.

Apesar da elegância teórica dos modelos físicos, estes apresentam sérias limitações. No caso particular dos canhões hidráulicos, além das incertezas quanto aos valores dos coeficientes de arraste, existem dificuldades de se estabelecer as condições iniciais das gotas (posição em relação ao aspersor, velocidade e ângulo da trajetória); no entanto, a literatura é rica em casos bem sucedidos da aplicação deste tipo de modelagem na simulação do desempenho de pequenos (Conceição \& Coelho, 2003) e médios aspersores (Tarjuelo et al., 1999; Montero et al., 2001; Playan et al., 2006).

Os modelos empíricos são desenvolvidos a partir de resultados de ensaios de distribuição espacial de água de aspersores realizados em diferentes condições de vento. Os modelos propostos por Seginer \& Kostrinsky (1975), Richards \& Weatherhead (1993), Han et al. (1994) e Gat \& Molle (2000) se encaixam nesta categoria.

O modelo de Richards \& Weatherhead (1993) vem recebendo a atenção de diversos pesquisadores interessados na simulação da distribuição de água de canhões hidráulicos operando em condições de vento (Granier et al., 2003; Newell, 2003; Smith et al., 2008; Prado, 2008; Oliveira, 2008). Por se tratar de um modelo cujos autores o classificam, em função da utilização de equações que tem forte embasamento teórico, como "semi-empírico", o modelo de Richards \& Weatherhead (1993) requer um número limitado de ensaios de campo para ajuste de seus parâmetros empíricos.

O modelo de Richards \& Weatherhead (1993) difere do modelo balístico convencional porque não se baseia apenas no efeito da deriva do vento sobre as gotas; este modelo assume que a transferência de movimento ao ar que circunda o jato, referida como fluxo de ar induzido, também afeta o alcance do canhão. Desta forma se observa, no sentido do vento, menor alteração no alcance do aspersor que a observada no sentido contra o vento porque, no sentido do vento, a ação da deriva é contraposta pela ação da interrupção do fluxo de ar induzido (a deriva tende a aumentar o alcance e a interrupção de fluxo tende a diminuí-lo) e, no sentido contra o vento, o efeito da deriva é somado ao efeito da interrupção do fluxo de ar induzido (ambos tendem a diminuir o alcance do jato).

Tendo em vista as vantagens decorrentes da utilização da modelagem matemática na simulação dos efeitos do vento sobre o padrão de distribuição espacial da água aplicada por canhões hidráulicos propôs-se, neste trabalho, avaliar, com base em ensaios de campo do canhão hidráulico da marca Plona, modelo RL250, a hipótese da existência de uma relação linear entre o raio de alcance de um canhão hidráulico e a velocidade do vento, que é assumida no modelo semi-empírico de Richards \& Weatherhead (1993).

\section{MATERIAL E MÉTODOS}

\section{O canhão Plona RL250}

Neste estudo foi utilizado um canhão hidráulico de reversão lenta da marca Plona, modelo RL250, que apresenta ângulo de inclinação do lançamento do jato de água de $24^{\circ}$, flange de acoplamento de $2 \frac{1}{2}$ " e giro proporcionado pelo impacto de um de seus dois braços oscilantes. Características operacionais deste canhão, obtidas em ensaios de laboratório, são dadas por Prado \& Colombo (2007).

\section{Ensaios de campo com vento}

Dados de distribuição espacial de água coletados em 53 ensaios do aspersor Plona RL250, realizados sob as diferentes condições de velocidade de vento e combinações de bocais e pressões de serviço mostradas na Tabela 1, foram utilizados neste trabalho.

Esses ensaios de campo foram realizados de acordo com a norma IS0 7749-2 (ISO, 1990), com auxílio de uma malha de 14 linhas por 14 colunas, de coletores pláticos, de $8 \mathrm{~cm}$ de diâmetro, regularmente espaçados $6 \mathrm{~m}$, perfazendo uma área de 84 x 84 m, em cujo centro foi instalado o canhão Plona RL250. O diâmetro dos coletores utilizados constituiu a única exceção à norma citada, a qual indica que os mesmos devem ter no mínimo $8,5 \mathrm{~cm}$ de diâmetro.

O sistema de eixos orientados (X, Y), utilizado nos ensaios de campo para determinar a posição dos coletores em relação ao Plona-RL250, está esquematizado na Figura 1. De 
Tabela 1. Velocidade média do vento e combinações de bocais e pressão de serviço registradas nos 53 ensaios de distribuição de água do aspersor Plona-RL250

\begin{tabular}{|c|c|c|c|c|c|c|c|c|c|c|}
\hline \multirow{3}{*}{$\begin{array}{l}\text { Bocal auxiliar } \\
\text { (mm) }\end{array}$} & \multicolumn{10}{|c|}{ Pressão de senviço (kPa) } \\
\hline & 392 & 490 & 392 & 490 & 392 & 490 & 392 & 490 & 392 & 490 \\
\hline & \multicolumn{10}{|c|}{ Velocidade média do vento durante cada ensaio $\left(\mathrm{m} \mathrm{s}^{-1}\right)$} \\
\hline \multirow[b]{2}{*}{7} & 4,96 & 4,72 & 2,95 & 3,13 & 5,32 & 5,83 & - & - & - & - \\
\hline & 2,96 & 3,45 & 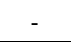 & 2,84 & 4,74 & 4,64 & - & - & - & - \\
\hline \multirow{3}{*}{6} & 1,76 & - & 2,79 & 1,83 & 3,57 & 3,39 & 2,43 & 2,71 & - & 2,87 \\
\hline & 0,97 & - & 2,61 & - & 2,54 & 1,34 & 1,73 & 2,04 & - & 2,74 \\
\hline & 0,96 & - & - & - & 2,01 & - & 1,00 & - & - & - \\
\hline \multirow{3}{*}{5} & 4,11 & 3,92 & 1,82 & 1,72 & 2,27 & 4,13 & - & - & - & - \\
\hline & 1,71 & 1,61 & 1,39 & 1,56 & - & 2,48 & - & - & - & - \\
\hline & - & - & 1,05 & - & - & - & - & - & - & - \\
\hline \multirow{3}{*}{ Sem Bocal } & 4,13 & 4,00 & 2,24 & 2,25 & 2,03 & 2,39 & - & - & - & - \\
\hline & 1,77 & 1,15 & 1,58 & 1,90 & - & 1,17 & - & - & - & - \\
\hline & - & - & - & 0,99 & - & - & - & - & - & - \\
\hline $\begin{array}{l}\text { Bocal Principal } \\
(\mathrm{mm})\end{array}$ & \multicolumn{2}{|c|}{14} & \multicolumn{2}{|r|}{16} & \multicolumn{2}{|c|}{18} & \multicolumn{2}{|c|}{20} & \multicolumn{2}{|c|}{26} \\
\hline
\end{tabular}

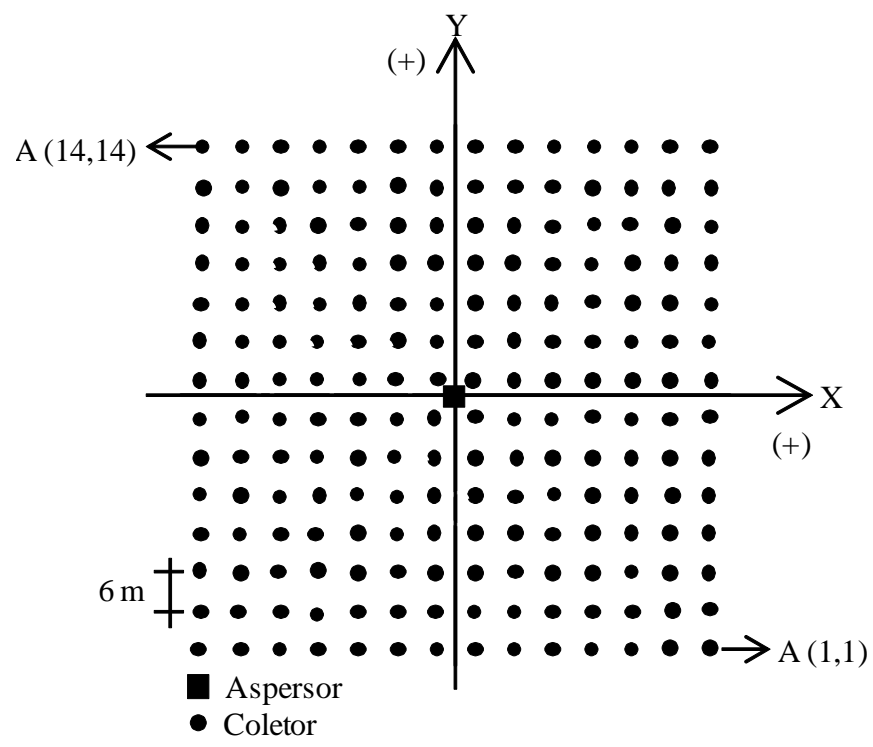

Figura 1. Sistema de eixos $(X, Y)$ utilizado nos ensaios de campo de distribuição de água realizados sob condições de vento

acordo com este sistema de eixos, o ponto de coordenadas $\mathrm{x}=0, \mathrm{y}=0$, representa a posição do Plona-RL250 e os pontos $\mathrm{x}=39 \mathrm{~m}, \mathrm{y}=-39 \mathrm{~m} \mathrm{e} \mathrm{x}=-39 \mathrm{~m}, \quad \mathrm{y}=39 \mathrm{~m}$ representam, respectivamente, os coletores cujas intensidades de precipitação foram armazenadas nos elementos $\mathrm{A}(1,1)$ e $\mathrm{A}(14,14)$ das matrizes em que foram registrados os dados de campo.

As coordenadas dos diversos coletores foram utilizadas juntamente com os valores das intensidades de precipitação $(\mathrm{Ii}, \mathrm{j})$, na determinação das coordenadas (XG, YG) do centro de gravidade (CG) da distribuição espacial da água observada em cada ensaio:

$$
X G=\frac{\sum_{i=1}^{i=14} \sum_{j=1}^{j=14} x_{i, j} \cdot I_{i, j}}{\sum_{i=1}^{i=14} \sum_{j=1}^{j=14} I_{i, j}}
$$

$$
Y G=\frac{\sum_{i=1}^{i=14} \sum_{j=1}^{i=14} y_{i, j} \cdot I_{i, j}}{\sum_{i=1}^{i=14 j=14} \sum_{j=1}}
$$

donde:

$\mathrm{X}_{\mathrm{i}, \mathrm{j}}$ - coordenada $\mathrm{x}$ do coletor $\mathrm{A}(\mathrm{i}, \mathrm{j})$

$\mathrm{Y}_{\mathrm{i}, \mathrm{j}}$ - coordenada y do coletor $\mathrm{A}(\mathrm{i}, \mathrm{j})$

\section{Determinações experimentais das dimensões da área molhada distorcida pelo vento}

As 53 matrizes contendo os dados da distribuição espacial da água observados nos ensaios realizados sob condições de vento, foram transformadas, com o auxílio do programa gráfico SURFER, versão 6.0 (Keckler, 1997), em mapas de superfície (Figura 2) que mostravam, em escala apropriada (1 $\mathrm{cm}=5,625 \mathrm{~m}$ ), a distribuição espacial das linhas de mesma intensidade de precipitação (desde $20,25 \mathrm{~mm} \mathrm{~h}^{-1}$ até $0,25 \mathrm{~mm}$ $\mathrm{h}^{-1}$ em intervalos de $5 \mathrm{~mm} \mathrm{~h}^{-1}$ ).

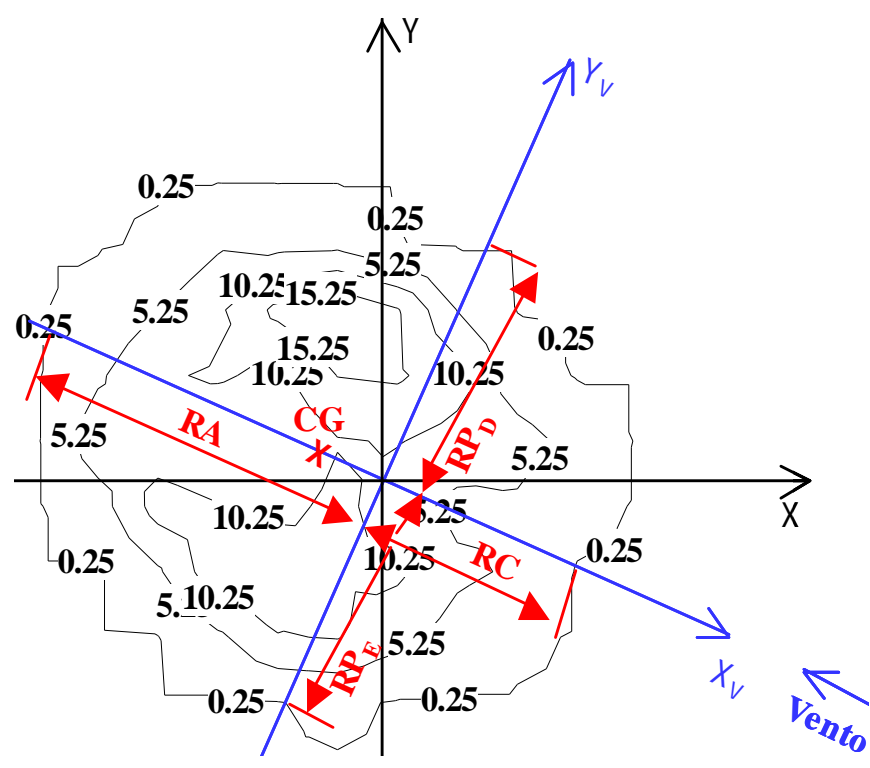

Figura 2. Sistema de eixos $(\mathrm{XV}, \mathrm{Yv})$ orientados em relação ao sentido predominante do vento

As dimensões da área molhada distorcida pelo vento foram determinadas em um sistema de eixos $\left(\mathrm{X}_{\mathrm{v}}, \mathrm{Y}_{\mathrm{v}}\right)$ orientados pelo sentido "predominante" do vento durante os ensaios (Figura 2). O sentido predominate do vento foi definido no sentido da reta que liga a posição de instalação do aspersor $(x=0, y=0)$ ao centro de gravidade $(x=X G$ e $y=Y G) d a$ distribuição espacial da água (Figura 2).

Conforme esquematizado na Figura 2, as medidas das distâncias do aspersor até a extremidade da área molhada pelo mesmo (definida pela posição da isoieta correspondente a uma intensidade de precipitação de $0,25 \mathrm{~mm} \mathrm{~h}^{-1}$ ) foram determinadas em quatro direções em relação ao sentido "predominante" do vento: (i) contra o vento (RC); (ii) a favor do vento (RA); (iii) perpendicular à direita $\left(\mathrm{RP}_{\mathrm{D}}\right) \mathrm{e}$ (iv) perpendicular à esquerda $\left(\mathrm{RP}_{\mathrm{E}}\right)$ do sentido do vento. Posteriormente, os valores da média das distâncias perpendiculares $\left[R P=\left(R_{D}+\right.\right.$ 
$\mathrm{RP}_{\mathrm{E}}$ ) / 2] e das distâncias contra (RC) e a favor (RA) do vento, foram expressas em termos de porcentagem (100RP/R, 100RC/R, 100RA/R) do raio de alcance sem vento (R). Os valores de raio de alcance sem vento $(\mathrm{R})$, correspondentes às diferentes condições operacionais do aspersor PlonaRL250 mostradas na Tabela 1, foram fornecidos pelo aplicativo Simulasoft, versão 3.0 (Prado, 2004).

\section{Modelagem das dimensões da área molhada}

A modelagem da distorção causada pelo vento, proposta por Richards \& Weatherhead (1993) utiliza do mesmo sistema de eixos $\left(X_{V}, Y_{V}\right)$, orientados em relação ao sentido do vento, esquematizado na Figura 2. Neste modelo, a relação entre as coordenadas $\left(\mathrm{x}_{\mathrm{v} 0}, \mathrm{y}_{\mathrm{v} 0}\right)$ de um ponto de impacto no solo que ocorre na condição de ausência de vento e as coordenadas $\left(\mathrm{x}_{\mathrm{v}}, \mathrm{y}_{\mathrm{v}}\right)$ de impacto deste mesmo ponto sob condições de vento não nulo (Figura 3) é dada pelas seguintes equações:

$$
\begin{gathered}
\mathrm{x}_{\mathrm{v}}=\mathrm{x}_{\mathrm{v} 0}-\left[\mathrm{A}+\mathrm{B} \cdot(\mathrm{r} / \mathrm{R})+\mathrm{C} \cdot(\mathrm{r} / \mathrm{R})^{2}\right] \cdot \mathrm{V}- \\
{\left[\mathrm{D} \cdot(\mathrm{r} / \mathrm{R})+\mathrm{E} \cdot(\mathrm{r} / \mathrm{R})^{2}+\mathrm{F} \cdot(\mathrm{r} / \mathrm{R})^{3}\right] \cdot \mathrm{V} \cdot \mathrm{S} \cdot \cos \theta} \\
\mathrm{y}_{\mathrm{v}}=\mathrm{y}_{\mathrm{v} 0}-\left[\mathrm{D} \cdot(\mathrm{r} / \mathrm{R})+\mathrm{E} \cdot(\mathrm{r} / \mathrm{R})^{2}+\mathrm{F} \cdot(\mathrm{r} / \mathrm{R})^{3}\right] \cdot \mathrm{V} \cdot \mathrm{S} \cdot \operatorname{sen} \theta
\end{gathered}
$$

em que:

A, B, C, D, E e F - constantes empíricas, s

$\mathrm{V}$ - velocidade do vento, $\mathrm{m} \mathrm{s}^{-1}$

e - ângulo de inclinação do lançamento do jato de água $\left(24^{\circ}\right)$.

$$
\begin{gathered}
r=\left(x_{v 0}{ }^{2}+y_{v 0}\right)^{2,5} \\
\cos \theta=x_{v 0} / r \\
\operatorname{sen} \theta=y_{v 0} / r \\
S=\left(\operatorname{sen}^{2} e \cdot \cos ^{2} \theta+\operatorname{sen}^{2} \theta\right)^{0,5}
\end{gathered}
$$

Nas Eqs. 3 e 4 os termos $\left(A+B(r / R)+C(r / R)^{2}\right)$ e $(D(r / R)+B(r /$ $\left.\mathrm{R})^{2}+\mathrm{C}(\mathrm{r} / \mathrm{R})^{3}\right)$ representam, respectivamente, o efeito da deriva

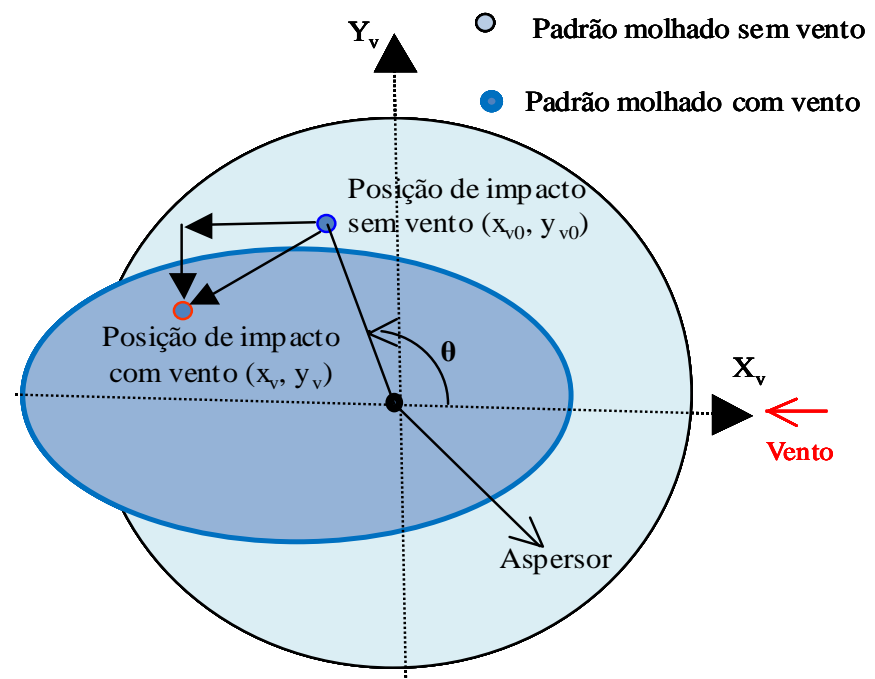

Figura 3. Deslocamento do ponto de impacto da água aplicada pelo aspersor devido à incidência de vento pelo vento e o efeito da interrupção do fluxo induzido de ar junto ao jato de água; nessas equações, a função (S) fornece o seno do ângulo entre o vetor velocidade do vento e a direção do jato, a partir do ângulo do jato no plano vertical (e; graus) e do ângulo entre o vetor vento e o jato de água no plano horizontal (è; graus).

A partir das Eqs. 3 e 4 é possível determinar as equações que regem as relações entre velocidade do vento e as distâncias até a extremidade da área molhada pelo aspersor, tomada nos sentidos (i) a favor do vento (RA), (ii) contra o vento (RC), (iii) perpendicular à direita $\left(\mathrm{RP}_{\mathrm{D}}\right)$ e (iv) perpendicular à esquerda $\left(\mathrm{RP}_{\mathrm{E}}\right)$ :

(i) No sentido a favor do vento ( $\left(\grave{\mathrm{e}}=180^{\circ}\right)$, a relação entre a distância até a extremidade da área molhada (RA) e a velocidade do vento, é estabelecida considerando-se, conforme esquematizado na Figura 4, os seguintes valores: $x_{v}=-R A$; $\mathrm{y}_{\mathrm{v} 0}=0 ; \mathrm{x}_{\mathrm{v} 0}=-\mathrm{R}$. Conseqüentemente, com $\mathrm{r}=\mathrm{R},(\mathrm{r} / \mathrm{R})=1$, cos $\grave{\mathrm{e}}=\mathrm{x}_{\mathrm{v} 0} / \mathrm{r}$ e $\mathrm{S}=0,407$ (dado pela Eq. 8), a Eq. 3 fornece:

$$
\begin{gathered}
-\mathrm{RA}=-\mathrm{R}-\left[\mathrm{A}+\mathrm{B} \cdot(1)+\mathrm{C} \cdot(1)^{2}\right] \cdot \mathrm{V}- \\
{\left[\mathrm{D} \cdot(1)+\mathrm{E} \cdot(1)^{2}+\mathrm{F} \cdot(1)^{3}\right] \cdot \mathrm{V} \cdot 0,407 \cdot(-1)} \\
\mathrm{RA}=\mathrm{R}+[(\mathrm{A}+\mathrm{B}+\mathrm{C})-(\mathrm{D}+\mathrm{E}+\mathrm{F}) \cdot 0,407] \cdot \mathrm{V}
\end{gathered}
$$

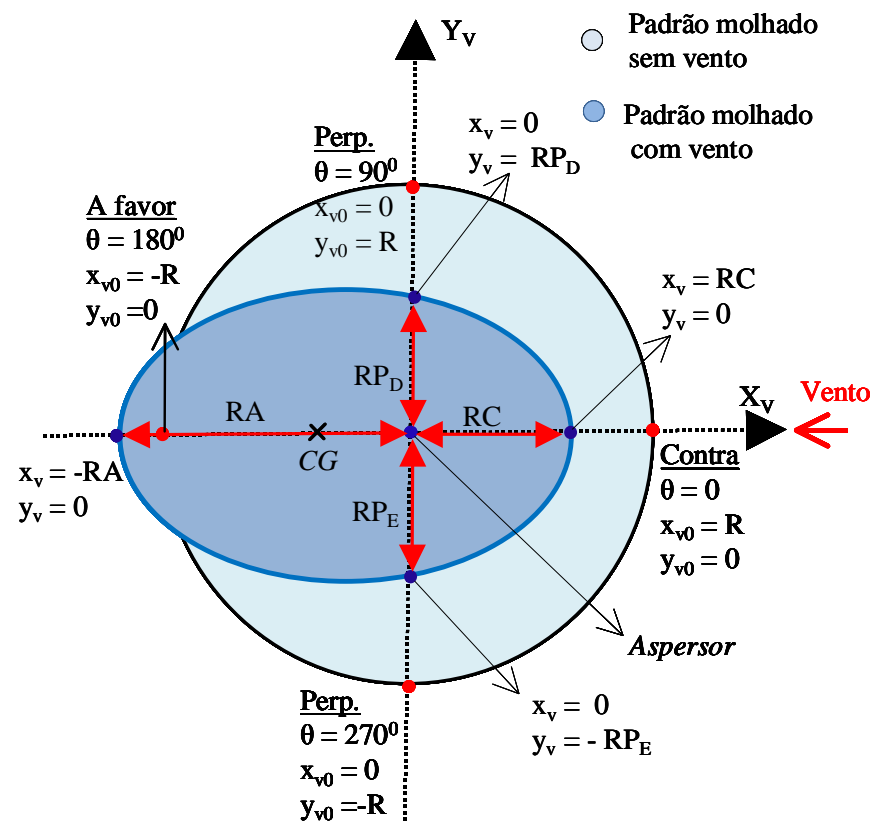

Figura 4. Distorção das dimensões do padrão de distribuição de água de um aspersor devido à incidência do vento

(ii) No sentido contra o vento $\left(\theta=0^{\circ}\right)$, a relação entre a distância até a extremidade da área molhada ( $\mathrm{RC})$, e a velocidade do vento (V), é estabelecida considerando-se, conforme esquematizado na Figura 4, os seguintes valores: $x_{v}=R C$; $\mathrm{y}_{\mathrm{v} 0}=0 ; \mathrm{x}_{\mathrm{v} 0}=\mathrm{R}$.

Conseqüentemente, com $r=R,(r / R)=1$, cos è $=x_{v o} / r$ e S = 0,407 (calculado pela Eq.8), a Eq. 3 fornece:

$$
\begin{gathered}
\mathrm{RC}=\mathrm{R}-\left[\mathrm{A}+\mathrm{B} \cdot(1)+\mathrm{C} \cdot(1)^{2}\right] \cdot \mathrm{V}- \\
{\left[\mathrm{D} \cdot(1)+\mathrm{E} \cdot(1)^{2}+\mathrm{F} \cdot(1)^{3}\right] \cdot \mathrm{V} \cdot 0,407 \cdot(1)} \\
\mathrm{RC}=\mathrm{R}-[(\mathrm{A}+\mathrm{B}+\mathrm{C})+(\mathrm{D}+\mathrm{E}+\mathrm{F}) \cdot 0,407] \cdot \mathrm{V}
\end{gathered}
$$


(iii) No sentido perpendicular à direita $\left(\theta=90^{\circ}\right)$, a distância até a extremidade da área molhada $\left(\mathrm{RP}_{\mathrm{D}}\right)$, em função da velocidade do vento (V), é calculada sem se levar em conta o efeito da deriva pelo vento; desta forma e conforme indicado na Figura 4, os seguintes valores são considerados: $y_{v}=R P_{D}$; $\mathrm{y}_{\mathrm{v} 0}=\mathrm{R} ; \mathrm{x}_{\mathrm{v} 0}=0$. Conseqüentemente, com $\mathrm{r}=\mathrm{R},(\mathrm{r} / \mathrm{R})=1$, sen $\theta=\mathrm{y}_{\mathrm{v} 0} / \mathrm{r}$ e $S=1$ (calculado pela Eq. 8), a Eq. 4 fornece:

$$
\begin{gathered}
\mathrm{RP}_{\mathrm{D}}=\mathrm{R}-\left[\mathrm{D} \cdot(1)+\mathrm{E} \cdot(1)^{2}+\mathrm{F} \cdot(1)^{3}\right] \cdot \mathrm{V} \cdot(1) \cdot(1) \\
\mathrm{RP}_{\mathrm{D}}=\mathrm{R}-(\mathrm{D}+\mathrm{E}+\mathrm{F}) \cdot \mathrm{V}
\end{gathered}
$$

(iv) No sentido perpendicular à esquerda $\left(\theta=270^{\circ}\right)$ a distância até a extremidade da área molhada $\left(\mathrm{RP}_{\mathrm{E}}\right)$, em função da velocidade do vento (V), é calculada sem se levar em conta o efeito da deriva. Desta forma e conforme indicado na Figura 4, os seguintes valores são considerados: $\mathrm{y}_{\mathrm{v}}=-\mathrm{RP}_{\mathrm{E}}$; $\mathrm{y}_{\mathrm{v} 0}=-\mathrm{R} ; \mathrm{x}_{\mathrm{v} 0}=0$. Conseqüentemente, com $\mathrm{r}=\mathrm{R},(\mathrm{r} / \mathrm{R})=1$, sen $\theta=\mathrm{y}_{\mathrm{v} 0} / \mathrm{r}$ e $S=1$ (calculado pela Eq. 8), a Eq. 4 fornece:

$$
\begin{gathered}
-\mathrm{RP}_{\mathrm{E}}=-\mathrm{R}-\left[\mathrm{D} \cdot(1)+\mathrm{E} \cdot(1)^{2}+\mathrm{F} \cdot(1)^{3}\right] \cdot \mathrm{V} \cdot(1) \cdot(-1) \\
\mathrm{RP}_{\mathrm{E}}=-[-\mathrm{R}+(\mathrm{D}+\mathrm{E}+\mathrm{F}) \cdot \mathrm{V}] \\
\mathrm{RP}_{\mathrm{E}}=\mathrm{R}-(\mathrm{D}+\mathrm{E}+\mathrm{F}) \cdot \mathrm{V}
\end{gathered}
$$

Quando as distâncias RA, RC, e RP $=\left(R_{D}+R P_{E}\right) / 2$ são expressas em termos de porcentagem do raio de alcance sem vento (R), as Eqs. 9b, 10b, 11b e 12c tomam as seguintes formas:

$$
\begin{aligned}
100 \cdot \frac{R A}{R} & =100+100 \cdot\left[\frac{(A+B+C)}{R}\right. \\
- & \left.0,407 \cdot \frac{(D+E+F)}{R}\right] \cdot V \\
100 \cdot \frac{R C}{R} & =100-100 \cdot\left[\frac{(A+B+C)}{R}\right. \\
& \left.+0,407 \cdot \frac{(D+E+F)}{R}\right] \cdot V \\
100 & \cdot \frac{R P}{R}=100-100 \cdot \frac{(D+E+F)}{R} \cdot V
\end{aligned}
$$

De acordo com essas equações, os coeficientes angulares da reta ajustada aos pares de valores experimentais, permitem estabelecer estimativas dos valores das somas dos coeficientes empíricos (100 (D/R+E/R+F/R) e $100(\mathrm{~A} / \mathrm{R}+\mathrm{B} / \mathrm{R}+\mathrm{C} / \mathrm{R}))$.

\section{RESULTADOS E DISCUSSÃO}

A viabilidade da utilização, no modelo de Richards \& Weatherhead (1993), de valores normalizados dos coeficientes empíricos (A/R, B/R, C/R, D/R, E/R e F/R) é demonstrada na Figura 5. A distribuição dos pontos experimentais demonstra a adequação da hipótese de uma relação linear entre raio de alcance e velocidade do vento.

Nas Figuras 5A, 5B e 5C, em cada curva correspondente às observações de campo foram considerados 53 valores de

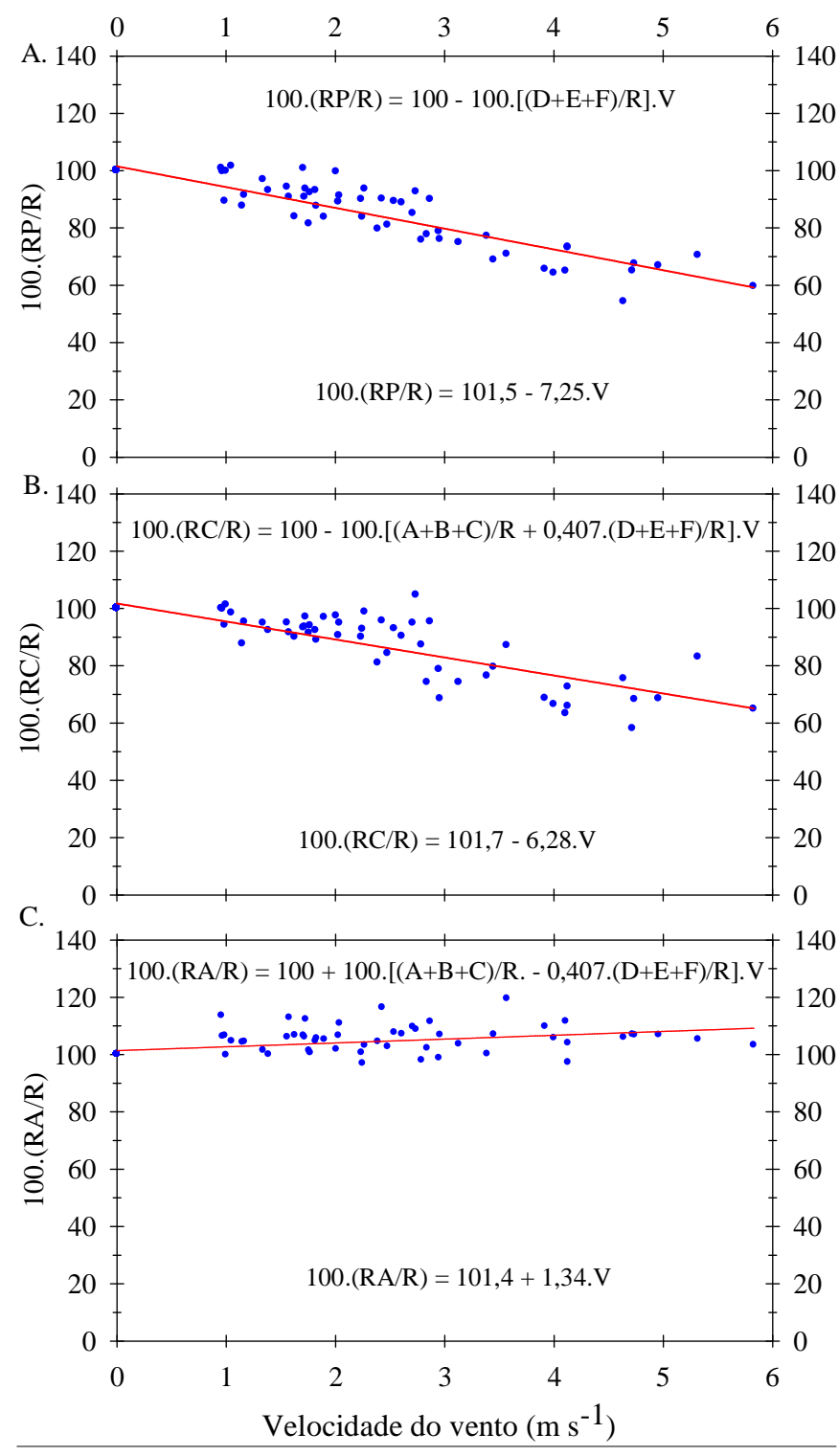

Figura 5. Distâncias relativas até a extremidade da área molhada, tomadas nas direções perpendicular $(A)$, contrária $(B)$ e a favor $(C)$ do vento, sob diferentes velocidades de vento

distância de alcance do jato, determinados em condições de vento, e 26 valores de raio de alcance, determinados através de simulações realizadas com o auxílio do Simulasoft, versão 3.0 (Prado, 2004); tais conjuntos de dados cobrem uma variação no valor do raio de alcance, sem vento, do aspersor Plona-RL250 que abrange desde 33,7 até 45,68 m.

A variação em função do valor da velocidade do vento, do valor médio $\left[R P=\left(R P_{D}+R P_{E}\right) / 2\right]$ das distâncias entre o aspersor e a extremidade da área molhada, tomadas em direções perpendiculares ao sentido do vento, é mostrada na Figura 5A. O bom ajuste dos dados observados no modelo linear $\left(r^{2}=0,850\right)$ e a proximidade entre o valor assumido pelo intercepto da reta ajustado ao valor teórico esperado (101,5 e 100), respectivamente, são indicadores da adequação da hipótese da linearidade. O confronto entre os termos da Eq. 13 e os termos da equação de ajuste obtida, que são mostrados na Figura 5A, fornece uma primeira estimativa quanto ao valor da soma dos fatores empíricos normalizados, utilizados no 
cálculo da redução do raio de alcance devido à interrupção do fluxo de ar induzido: (D/R+E/R+F/R $=0,0725 \mathrm{~s} \mathrm{~m}^{-1}$ ).

A variação em função do valor da velocidade do vento, do valor da distância relativa $(\mathrm{RC} / \mathrm{R})$ entre o aspersor e a extremidade da área molhada, tomada em sentido contrário ao vento, é mostrada na Figura 5B. O bom ajuste dos dados observados no modelo linear $\left(\mathrm{r}^{2}=0,737\right)$ e a proximidade entre o valor assumido pelo intercepto da reta ajustado ao valor teórico esperado (101,7 e 100) são indicadores da adequação da hipótese de linearidade. O confronto entre os termos da Eq. 14 e os termos da equação da reta de ajuste obtida, mostrados na Figura 5B, juntamente com o valor previamente estimado de $(\mathrm{D} / \mathrm{R}+\mathrm{E} / \mathrm{R}+\mathrm{F} / \mathrm{R})$ permite estabelecer uma primeira estimativa do valor da soma dos fatores empíricos normalizados para cálculo da deriva pelo vento: $(A / R+B / R+C / R=0,0333$ $\left.\mathrm{s} \mathrm{m}^{-1}\right)$.

A variação em função do valor da velocidade do vento, do valor da distância relativa (RA/R) entre o aspersor e a extremidade da área molhada, tomada no sentido a favor do vento, é mostrada na Figura 5C. No caso da Figura 5C, o coeficiente de determinação da reta de ajuste obtida não foi tão bom quanto os anteriores $\left(r^{2}=0,215\right)$, mas a proximidade entre o valor assumido pelo intercepto da reta ajustado e valor teórico esperado (101,4 e 100) foi mantida. De acordo com a Eq. 9b, a forma ascendente da reta de ajuste obtida indica que, na direção do vento, o efeito de redução do raio de alcance, provocado pela interrupção do fluxo induzido de ar, é pouco menor que o efeito de alongamento do raio provocado pela deriva do vento.

Com base no confronto entre os termos da Eq. 13 e os termos da equação de ajuste obtida, mostrados na Figura 5C e juntamente com o valor previamente estimado de (D/R+E/ $\mathrm{R}+\mathrm{F} / \mathrm{R})$, é possível estabelecer uma segunda estimativa para o valor da soma dos fatores empíricos normalizados utilizados no cálculo da deriva pelo vento: $(A / R+B / R+C / R)=0,0429$ $\mathrm{s} \mathrm{m}^{-1}$. Esta segunda estimativa, apesar de ser pouco maior que a primeira, é próxima o suficiente para permitir que seja utilizada uma estimativa baseada na média das duas determinações anteriores $\left(\mathrm{A} / \mathrm{R}+\mathrm{B} / \mathrm{R}+\mathrm{C} / \mathrm{R}=0,0381 \mathrm{~s} \mathrm{~m}^{-1}\right)$.

Os resultados apresentados na Figura 5, além de permitirem demonstrar a adequação da hipótese da linearidade entre raio de alcance e a velocidade do vento e fornecerem estimativas quanto ao valor assumido pelos coeficientes empíricos de Richards \& Weatherhead (1993), permitem inferir que: (i) para cada incremento de $1 \mathrm{~m} \mathrm{~s}^{-1}$ na velocidade do vento, o raio de alcance do aspersor, na direção perpendicular à linha de ação do vento (RP, na Figura $5 \mathrm{~A}$ ), sofre uma redução de $7,3 \%$, em relação ao valor sem vento $(\mathrm{R})$; (ii) para cada incremento de $1 \mathrm{~m} \mathrm{~s}^{-1}$ na velocidade do vento, o raio de alcance do aspersor, tomado no sentido contrário à linha de ação do vento (RC, na Figura 5B), sofre uma redução de 6,3\%, em relação ao valor sem vento (R); (iii) para cada incremento de $1 \mathrm{~m} \mathrm{~s}^{-1}$ na velocidade do vento, o raio de alcance do aspersor, tomado no sentido da linha de ação do vento (RA - Figura 5C), sofre um acréscimo de 1,3\%, em relação ao valor sem vento $(\mathrm{R})$.

A ordem de grandeza das variações no raio de alcance do aspersor Plona-RL250, causadas pelo vento, e observadas neste estudo, é bastante próxima daquelas obtidas por Richards \& Weatherhead (1993), com o aspersor Nelson P150, operando com bocal de $25,4 \mathrm{~mm}$ e ângulo de inclinação do jato de $24^{\circ}$ que encontraram para cada incremento de $1 \mathrm{~m} \mathrm{~s}^{-1}$ na velocidade do vento, reduções de alcance nas direções perpendiculares e contra o vento, da ordem de $6,4 \%$ do valor do raio sem vento e, na direção do vento, aumento de alcance da ordem de $1,2 \%$.

\section{CONCLUSÃO}

A hipótese da existência de uma relação linear entre o raio de alcance de um canhão hidráulico e a velocidade do vento foi comprovada.

\section{LITERATURA CITADA}

Azevedo, H. J.; Bernardo, S.; Ramos, M. M.; Sediyama, G. C.; Cecon, P. R. Influência de fatores climáticos e operacionais sobre a uniformidade de distribuição de água, em um sistema de irrigação por aspersão de alta pressão. Revista Brasileira de Engenharia Agrícola e Ambiental, v.4, n.2, p.152-158, 2000.

Carrión, P.; Tarjuelo, J. M.; Montero J. SIRIAS: A simulation model for sprinkler irrigation. Part I: Description of model. Irrigation Science, v.20, n.2, p.73-84, 2001.

Conceição, M. A. F.; Coelho, R. D. Simulating wind effect on microsprinkler water distribution. Scientia Agricola, v.60, n.2, p.205-209, 2003.

Gat, Y. L.; Molle, B. Model of water applications under pivot sprinkler. I Theoretical Gruonds. Journal of Irrigation and Drainage Engineering, v.126, n.6, p.343-347, 2000.

Granier, J.; Molle, B.; Deumier, J. M. IRRIPARC-Part 1: Modeling spatial water distribution under a sprinkler in windy conditions. ICID International Workshop, Montpellier, 2003. Proceedings......Aix en Provence: Cemagref, 2003. p.14-19.

Han, S.; Evans, R. G.; Kroeger, M. W. Sprinkler distribution patterns in windy conditions. Transactions of the American Society of Agricultural Engineers, v.37, n.5, p.1481-1489, 1994.

ISO - International Organization for Standardization. ISO 77492: Irrigation equipment: Rotating sprinklers: Part 2: Uniformity of distribution and test methods. Switzerland: International Organization for Standardization, 1990. 6p.

Keckler, D. Surfer for windows: version 6 User's Guide - Contouring and 3D Surface Mapping. Golden: Golden Software, 1997. 540p.

Montero J.; Tarjuelo, J. M.; Carrión, P. SIRIAS: A simulation model for sprinkler irrigation. Part II: Calibration and validation of the model. Irrigation Science, v.20, n.2, p.85-98, 2001.

Newell, G. Traveling gun simulation model TRAVGUN: User's manual and technical documentation. Toowoomba: National Centre for Engineering in Agriculture-NCEA. University of Southern Queensland-USQ, 2003. 89p.

Oliveira, H. F. E. Modelagem semi-empírica da distribuição de água de aspersores autopropelidos sob diferentes condições de vento. Lavras: UFLA, 2008. 74p. Dissertação Mestrado 
Ortega, J. F. A.; Tarjuelo, J. M. M.; de Juan, J. A. V.; Carrion, P. P. Uniformity distribution and its economic effect on irrigation management in semiarid zones. Journal of Irrigation and Drainage Engineering, v.130, n.4, p.257347, 2004.

Playan, E.; Zapata, N.; Faci, J. M.; Tolosa, D.; Lacuerva, J. L.; Pelegri, J. Salvador, R.; Sanches, I.; Lafita, A. Assessing sprinkler irrigation uniformity using a ballistic simulation model. Agricultural Water Management, v.84, n.1, p.89-100. 2006.

Prado, G. Aplicativo computacional para simulação da distribuição de água pelo aspersor Plona-RL300 em sistemas autopropelidos de irrigação. Lavras: UFLA, 2004. 86p. Dissertação Mestrado

Prado, G. Modelagem da aplicação de água com canhões hidráulicos sob diferentes condições de vento. Lavras: UFLA, 2008. 121p. Tese Doutorado
Prado, G.; Colombo, A. Análise da uniformidade de aplicação de água pelo aspersor Plona-RL250 em sistemas autopropelidos de irrigação. Irriga, v.12, n.2, p.249-262, 2007.

Richards, P. J.; Weatherhead, E. K. Prediction of raingun application patterns in windy conditions. Journal of Agricultural Engineering Research. v.54, p.281-291. 1993.

Seginer, I.; Kostrinsky, N. Wind, sprinkler pattern and system design. Journal of Irrigation and Drainage Engineering, v.101, n.4, p.251-264, 1975.

Smith, R. J.; Gillies, M. H.; Newell, G.; Foley, J. P. A decision support model for travelling gun irrigation machines, Biosystems Engineering, v.100, n.1, p.126-136, 2008.

Tarjuello, J. M.; Monteiro, J.; Carrion, P. A.; Honrubia, F. T.; Calvo, M. A. Irrigation uniformity with medium size sprinklers part II: Influence of wind and other factors on water distribution. Transactions of the American Society of Agricultural Engineers, v.42, n.3, p.677-689, 1999. 